\title{
Visão de Cores no Primeiro Ano de VidA
}

\author{
Dora Fix Ventura ${ }^{1}$ \\ Instituto de Psicologia - USP
}

\begin{abstract}
Procedimentos comportamentais que permitem inferir capacidades sensoriais ou perceptuais em bebês e crianças baseiam-se, na sua maior parte, na descoberta seminal feita no fim da década de 1950 pelo psicólogo Robert Fantz. Desde o nascimento, há uma preferência do bebê por dirigir o olhar para estímulos complexos a fixá-lo em cenas monótonas. Baseado no novo conhecimento, o grupo liderado por Davida Teller na Universidade de Washington desenvolveu uma metodologia de avaliação psicofísica da visão em bebês, o que possibilitou grande avanço no estudo do desenvolvimento e da maturação de várias funções visuais básicas, como acuidade visual, visão de cores, visão de contrastes, estereopsia $e$ visão de movimento. A presente revisão examina essa literatura, mostrando como é avaliada a visão em bebês e o que se conhece até o momento sobre a capacidade dos recém-nascidos de ver cores.
\end{abstract}

Descritores: Discriminação de cores. Desenvolvimento. Bebês.

\section{Características da visão de cores}

\begin{abstract}
A s cores que vemos não estão nos objetos, dependem de propriedades do nosso sistema nervoso. A detecção de luz se dá pela atividade de células especializadas que transformam a energia da luz em resposta neural. Essas células são os fotorreceptores da retina - os cones e bastonetes - e sua capacidade de detectar luz varia ao longo do espectro eletromagnético, do vermelho ao violeta. A visão diurna, com a qual enxergamos as cores, é mediada pelos cones, enquanto a noturna é mediada pelos bastonetes. Na retina humana exis-

1 Docente do Departamento de Psicologia Experimental e Núcleo de Neurociências e Comportamento do Instituto de Psicologia - USP. Endereço eletrônico: dventura@usp.br
\end{abstract}

Psicologia USP, 2007, 18(2), 83-97. 
tem três diferentes tipos de cones, cada um dos quais absorve luz em praticamente todo o espectro visível para o ser humano (400 a $700 \mathrm{~nm}$ ), mas difere dos outros na região em que tem mais probabilidade de absorver luz. Os três diferentes tipos de cones da retina humana são maximamente sensíveis, respectivamente, a comprimentos de onda longos (cone L), médios (cone $\mathrm{M}$ ou verde) e curtos (cone C). São chamados de cones vermelhos, verdes e azuis, mas erroneamente, pois não têm essas cores e, principalmente no caso do primeiro deles, o pico de absorção situa-se no amarelo, não no vermelho. As diferenças entre esses tipos de cone decorrem da capacidade de absorção de luz da molécula de pigmento fotossensível (opsina) que existe em cada um deles. As opsinas são proteínas cuja expressão na retina é geneticamente determinada e depende de diferentes genes. Destes, dois são os responsáveis pelas opsinas L e M e estão localizados no cromossomo X, o terceiro é responsável pela opsina C e está no cromossomo 7. Na ausência de um desses genes, há cegueira parcial para cores (popularmente chamada de daltonismo). A mais freqüente é a falta ou o defeito na expressão dos genes para as opsinas L e M, o que causa prejuízo na visão do verde-vermelho. Isso ocorre em cerca de $5 \%$ da população geral, mas como se trata de um defeito ligado ao cromossomo X, atinge muito mais os homens, ocorrendo em 8 a $10 \%$ da população masculina. Na total ausência de uma das opsinas, o indivíduo é dicromata, ou seja, vê cores de forma incompleta: a visão humana normal é tricromata.

Para ver as cores, precisamos não só que existam os fotorreceptores que acabamos de descrever, mas que também exista uma rede neural ligada a esses fotorreceptores, comparando suas respostas às diferentes regiões do espectro. É a partir da interação entre os canais neurais ligados a cada um dos tipos de cones que existe a codificação da informação sobre cor. A visão noturna é mediada por um só tipo de célula - os bastonetes - e por esse motivo é acromática, ou seja, desprovida da capacidade de ver cores.

Teorias psicofísicas propuseram que a visão de cores é mediada pela recombinação da atividade dos três tipos de cones em três canais: um acromático, que reflete a atividade somada dos três tipos de cones, e dois cromáticos, dos quais um verde/vermelho, em que os canais neurais correspondentes aos cones L e M interagem em oponência e outro azul/amarelo, em que a oponência se dá entre o canal neural originado no cone $\mathrm{C}$ e os canais $\mathrm{L}$ e $\mathrm{M}$ em sinergia (Boynton, 1979). Esses três canais são constituídos, respectivamente, 


\section{Visão de Cores no Primeiro Ano de Vida}

pelas vias magno-, parvo- e koniocelular, identificadas fisiológica e morfologicamente (Lee, 1997; Merigan \& Maunsell, 1993).

Estudos recentes do desenvolvimento dos fotorreceptores da retina, com técnicas imunocitoquímicas, revelaram que a expressão dessas opsinas na retina pode ser detectada no desenvolvimento fetal, mas não ocorre simultaneamente para os três tipos. As opsinas dos cones $\mathrm{S}$ (azuis) aparecem na fóvea e em torno dela, entre a $10^{\mathrm{a}}$ e $11^{\mathrm{a}}$ semana de vida fetal, enquanto as dos cones L (vermelho) e M (azul) aparecem mais tarde, entre a $14^{\mathrm{a}}$ e $15^{\mathrm{a}}$ semanas. O desenvolvimento dos cones na retina também é diferente do centro para a periferia: enquanto os cones S cobrem $90 \%$ da retina na $19^{\mathrm{a}}$ semana fetal, os L e M só chegam à periferia entre a 34a e 37a semanas (Xiao \& Hendrickson, 2000). Assim, pelo menos em função do aparecimento dos diferentes tipos de cones, o sistema do azul se desenvolve antes do sistema do verde-vermelho.

Durante o desenvolvimento, há não só a necessidade da formação dos fotorreceptores, como a da constituição de uma complexa rede de interações para que exista visão de cores. Embora os diferentes cones já estejam presentes no nascimento, o desenvolvimento da capacidade de ver cores é lento, só se completando entre 18 e 20 anos de idade, quando a capacidade de discriminação de cores no ser humano é máxima e começa lentamente a decrescer, até o fim da vida (Knoblauch, Vital-Durand, \& Barbur, 2001; Ventura et al., 2002).

\section{Como se avalia a visão de cores em bebês?}

A discriminação de cores é a capacidade de distinguir entre estímulos visuais que diferem apenas pela composição de comprimentos de onda. O correlato psicofísico dessa dimensão é o matiz. Para que se possa afirmar que há visão de cores, a discriminação deve independer de outros atributos do estímulo visual, como intensidade, área, bordas, textura, etc. Um dos atributos que mais comumente impede conclusões inequívocas sobre a discriminação de cores em bebês é a intensidade ou luminância ${ }^{2}$, cujo correspondente sub-

2 A luz é medida em unidades fotométricas, que levam em consideração a sensibilidade humana aos diferentes comprimentos de onda expressa na função fotópica de sensibilidade espectral. Luminância é o correspondente fotométrico da intensidade, um termo 
jetivo é o brilho. Foi apenas na década de 1970 que os pesquisadores fizeram esse controle e conseguiram mostrar inequivocamente que bebês eram capazes de fazer discriminações baseadas apenas na cor (Bornstein, 1975; Kessen \& Bornstein, 1978; Peeples \& Teller, 1975; Schaller, 1975). Antes disso, as conclusões sobre capacidade de discriminar cores em bebês podiam estar contaminadas por essa variável, ou seja, as discriminações atribuídas a cor poderiam ter sido feitas com base na intensidade.

Nesses primeiros esforços, estímulos heterocromáticos eram ajustados em brilho usando-se valores de sensibilidade espectral de adultos, o que poderia ser aceitável como primeira aproximação, pois são semelhantes aos dos bebês (Dobson, 1976; Peeples \& Teller, 1978; Teller, Peeples \& Sekel, 1978), mas não é o ideal. Os estímulos cromáticos precisariam ser muito cuidadosamente igualados quanto ao brilho para que se pudesse chegar a conclusões seguras de que a discriminação se baseia apenas na cor e, para esses ajustes, seria necessário conhecer a função de sensibilidade espectral fotópica do bebê, ou seja, a sensibilidade do sistema de visão diurno a diferentes comprimentos de onda. Essa lacuna foi preenchida com experimentos realizados pelo grupo de Davida Teller, que determinou a curva de sensibilidade espectral em recémnascidos, com métodos comportamentais (Teller et al., 1978).

Além do problema da equiparação de intensidades, existe o problema da forma como se vai medir a discriminação de cores. Como fazer com que o bebê nos comunique a sua capacidade de ver cores?

Para avaliar a visão de cores em adultos, existem inúmeros testes, mas todos dependem de compreensão de instruções verbais e de repertórios de comportamentos que não existem no recém-nascido, nem mesmo na criança até cerca de 5 anos de idade. Uma forma de obter informação sobre a visão de cores, ou sobre o potencial neurológico para essa capacidade, é recorrer a respostas fisiológicas a estímulos cromáticos. As respostas fisiológicas podem ser obtidas na retina, com o Eletrorretinograma (ERG), ou no córtex occipital, com o Potencial Visual Evocado (PVE). Entretanto, é só através do comportamento que se pode comprovar que a capacidade de ver cores existe e é usada pelo indivíduo. Descreveremos a seguir os resultados obtidos com métodos

genérico que não reflete o sistema de medida. A luminância é medida em candelas $/ \mathrm{m}^{2}$ ou unidades equivalentes. 
eletrofisiológicos, assim como os engenhosos métodos comportamentais desenvolvidos por psicólogos para investigar a visão de cores em bebês.

\section{Visão de cores avaliada com métodos eletrofisiológicos}

O PVE é uma resposta visual cortical, obtida estimulando-se o sistema visual com pulsos de luz, padrões em branco e preto ou cores. É registrado como um eletroencefalograma (EEG) das áreas occipitais, no qual, fazendo-se a soma de várias respostas a pulsos de luz, a atividade irregular do EEG desaparece, pois não se repete de um registro para outro, e pode-se ver a resposta aos pulsos de luz, que se repete a cada estimulação. Quanto maior o número de repetições, maior a eliminação das irregularidades do EEG e melhor a caracterização da resposta ao pulso de luz. Podem ser também usados padrões de outros tipos para o registro do PVE. Um dos mais usados é o padrão reverso de grades em branco e preto, nos quais a luminância das listras é invertida de branco para preto num ritmo regular. A cada troca é gerada uma resposta cortical.

Em infantes humanos, demonstrou-se que já na $4^{\text {a }}$ semana de vida, os cones L ou M são capazes de gerar, separadamente, respostas de PVE, o que sugere que ambos os tipos de cones são funcionais nessa idade (Knoblauch, Bieber, \& Werner, 1998). Entretanto, essa é uma condição necessária mas não suficiente para a visão de cores. Para que haja visão de cores, não só os diferentes tipos de cones precisam ser funcionais, mas o restante da rede neural precisa ser capaz de diferenciar entre cor e luminância. Entre 6 e 15 semanas de vida, os potenciais visuais evocados de bebês não diferem nas respostas a estímulos de luminância e a cores, mas a partir da $15^{\text {a }}$ semana de vida, a morfologia da resposta de luminância muda, principalmente nos primeiros 100 ms, adquirindo um novo componente, que tenderá a dominar a resposta adulta. A partir dessa idade, portanto, parece haver o potencial neural para uma resposta cromática diferente da resposta de luminância (Morrone, Fiorentini, \& Burr, 1996). Anteriormente, o mesmo grupo havia sugerido que a visão de luminância e a visão de cores têm desenvolvimentos independentes com ritmos diferentes. Essa conclusão baseou-se na constatação de que a resposta a um estímulo com $20 \%$ de contraste de luminância era confiável em recémnascidos de 7-8 semanas de vida, mas que não havia nessa idade respostas a 


\section{Dora Fix Ventura}

estímulos puramente cromáticos, ou seja, estímulos que não diferem quanto à luminância (eqüiluminantes) e só diferem quanto à cromaticidade (Morrone, Burr \& Fiorentini, 1993).

Uma técnica mais recente é o PVE de varredura, originariamente usado para medir acuidade visual de resolução de grades (ver artigo de Salomão neste volume) e usada também para medir sensibilidade ao contraste (ver Oliveira, Costa, De Souza \& Ventura, 2004, para primeiros registros de sensibilidade ao contraste por PVE de varredura em bebês feitos no Brasil) ou outras funções visuais. No PVE de varredura, enquanto se registra a atividade elétrica nas áreas occipitais da mesma forma que no PVE tradicional, a estimulação é continuamente modificada ao longo da variável que se quer investigar (freqüência espacial, contrate ou outra) até se obter um limiar. Os limiares são obtidos em poucos minutos, desde que o sujeito testado esteja alerta e olhando para o monitor de vídeo. Esse método foi empregado para comparar visão acromática e cromática, em funções de sensibilidade ao contraste de luminância e verde-vermelho eqüiluminante. Verificou-se que em bebês de 8 semanas de vida existe resposta confiável ao contraste, mas com baixa sensibilidade, e que há aumento da sensibilidade até a idade adulta. O aumento da sensibilidade é menor para grades acromáticas (de $0.64 \log$ ) do que para as cromáticas $(0.91 \mathrm{log})$ (Kelly, Borchert, \& Teller, 1997; Kelly \& Chang, 2000). Esses estudos mostraram, ainda, que a faixa de frequiências espaciais visíveis também aumenta com a idade, mas de forma semelhante para grades acromáticas e cromáticas, e que esses aumentos correspondem às predições baseadas no crescimento do tamanho de olho e no aumento de densidade de fotorreceptores na fóvea.

Em conclusão, o PVE permitiu verificar que a partir da $8^{\mathrm{a}}$ semana de vida existe o potencial neurológico para a visão de cores verde e vermelha em bebês humanos e que, por volta da $15^{\text {a }}$ semana de vida, há uma diferença clara no PVE cromático em relação ao acromático. Ao longo do desenvolvimento, a função de contraste cromático melhora em sensibilidade e abrangência espacial.

\section{Visão de cores avaliada por técnicas comportamentais}

Procedimentos comportamentais que permitem inferir capacidades sensoriais ou perceptuais em bebês e crianças baseiam-se, na sua maior parte, 
na descoberta seminal feita na década de 1950 pelo psicólogo Robert Fantz: desde o nascimento, há uma preferência por dirigir o olhar para estímulos complexos a fixá-lo em cenas monótonas (Fantz, 1956, 1958, 1960, 1961, 1963, 1964, 1965; Fantz \& Miranda, 1975; Fantz \& Nevis, 1967; Fantz \& Ordy, 1959; Fantz, Ordy, \& Udelf, 1962). Essa descoberta forneceu a base metodológica para estudar percepção desde o início da vida e constituiu o ponto de partida para uma nova compreensão a respeito das habilidades e competências do recém-nascido. Por meio desses estudos, foi possível constatar que a capacidade perceptual no nascimento é muito mais complexa e organizada do que se supunha anteriormente.

Baseada no novo conhecimento, de que o bebê apresenta resposta inata de dirigir o olhar a estímulos complexos, Davida Teller e seus colaboradores (Peeples \& Teller, 1975) desenvolveram uma metodologia de avaliação psicofísica da visão em bebês que possibilitou grande avanço no estudo do desenvolvimento e da maturação de várias funções visuais básicas, como acuidade visual, visão de cores, visão de contrastes, estereopsia e visão de movimento.

O procedimento desenvolvido por Teller foi usado inicialmente para medir acuidade visual. Consistia no julgamento da direção do olhar do bebê por um observador numa situação em que dois estímulos eram apresentados lado a lado - método psicofísico chamado olhar preferencial de escolha forçada. No caso de medida da acuidade visual, esses estímulos eram uma grade em branco e preto e um cinza homogêneo. Diante desses dois estímulos, o bebê dirigia o olhar à grade, manifestando sua preferência pelo mais complexo dos dois estímulos. A resposta ocorria sempre que a grade continha listras largas (baixa freqüência espacial), mas, quando as listras diminuíam de largura (frequiência espacial mais alta), começava a diminuir a preferência pela grade, e chegava-se a uma freqüência espacial em que a preferência desaparecia. A interpretação era de que o bebê deixava de manifestar preferência porque não estava mais vendo as listras da grade, por serem mais finas do que sua acuidade permitia decodificar; deveria estar vendo um cinza homogêneo igual ao do estímulo de comparação. Assim, a frequiência espacial em que a preferência desaparecia era considerada correspondente ao limiar de resolução para aquele bebê. Esse procedimento deu origem ao teste dos Cartões de Acuidade de Teller, hoje amplamente usados na clínica em todo o mundo, e para o qual 
contamos com normas feitas no Brasil (Peeples \& Teller, 1975; Salomao \& Ventura, 1993, 1995).

O procedimento psicofísico mais utilizado nos estudos de visão de cores baseou-se no estudo pioneiro do grupo de Davida Teller (Peeples \& Teller, 1975), no qual o ponto central era demonstrar a existência de visão de cores na ausência de pistas de brilho. O estímulo era um campo vermelho apresentado sobre um fundo branco e comparado com um estímulo idêntico, que continha apenas o fundo branco, apresentado ao lado. Usando a metodologia do olhar preferencial de escolha forçada, um observador decidia para qual dos estímulos o bebê, confortavelmente sentado no colo de um adulto, estava olhando. Com essa técnica foram testados infantes de 2 meses, com estímulos vermelhos de várias intensidades comparados com o fundo branco equiparado em brilho com o vermelho para o adulto. Os bebês responderam significativamente mais para os estímulos vermelhos em todas as luminâncias, o que permitiu concluir que eles têm alguma forma de visão de cores.

Posteriormente, o mesmo procedimento foi usado por Teller e colaboradores (Hammer, Alexander, \& Teller, 1982) em bebês de 1 a 3 meses, para testar uma gama variada de cores, verificando inclusive se os bebês conseguiam fazer discriminações de Rayleigh ${ }^{3}$ e discriminações ao longo do eixo tritan (azul-amarelo), com a finalidade de identificar possíveis defeitos congênitos na visão de cores (daltonismo). Os bebês de 1 mês não conseguiam fazer essas discriminações, mas os de 2 meses em diante conseguiam. A mostra os resultados obtidos, apresentados no espaço de cromaticidade de MacLeod e Boynton (1979), no qual os dois eixos representam, respectivamente, a ativação dos sistemas oponentes verde-vermelho (protan-deutan) e azul-amarelo (tritan). As linhas verticais tracejadas mostram a região em que os bebês não conseguiram ou tiveram maior dificuldade de discriminar cores. Isso ocorreu na região tritan, em concordância com outros estudos, sugerindo maturação mais lenta do sistema de oponência azul-amarelo. Figura 1.

3 A equação de Rayleigh estabelece a proporção de verde e vermelho numa mistura dessas duas cores que é considerada de aparência igual a um amarelo monocromático (usualmente de $590 \mathrm{~nm}$ ). Constitui o método mais exato para diferenciar protanomalia de deuteranomalia (respectivamente, perda em discriminações cromáticas no canal do cone L e no canal do cone $\mathrm{M}$ ). 


\section{Visão de Cores no Primeiro Ano de Vida}

Data from Teller, Peeples \& Sekel, 1978

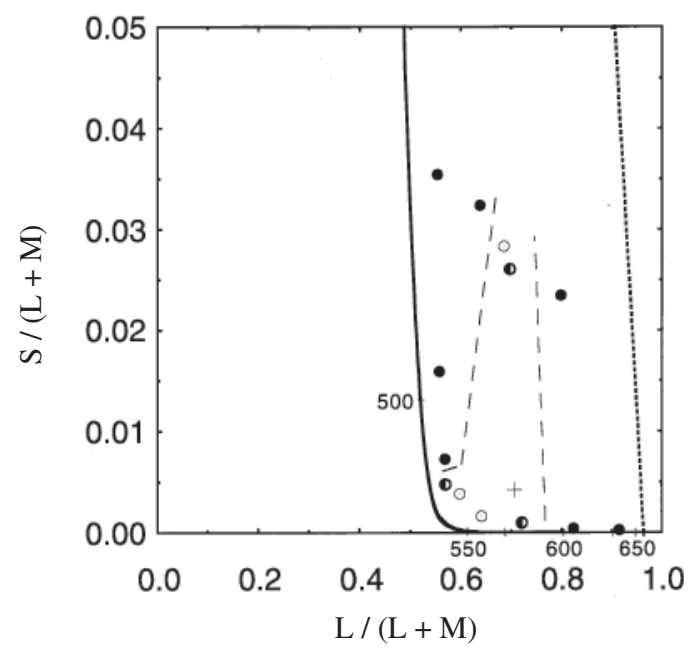

Figura 1. Dados de discriminação de cores em infantes de 2 meses apresentados no espaço de cromaticidade MacLeod and Boynton. no qual o eixo das abcissas representa a ativação do sistema verde-vermelho $(\mathrm{L} / \mathrm{L}+\mathrm{M})$ e a ordenada, a ativação do sistema azul-amarelo $(\mathrm{S} / \mathrm{L}+\mathrm{M})$. A linha cheia mostra o lócus espectral com números indicando estímulos monocromáticos de 500 a 650 $\mathrm{nm}$. A linha pontilhada (à direita) conecta os extremos do espectro (cores não-espectrais). Os círculos mostram as cromaticidades dos estímulos que foram testados contra um fundo branco (cruz). Os círculos fechados correspondem a discriminações bem sucedidas; os círculos abertos, a falhas de discriminação, e os círculos semiabertos, a casos em que alguns infantes conseguiram discriminar e outros falharam. As linhas tracejadas delimitam todos os casos de insucesso na discriminação, correspondendo a uma linha vertical. Figura modificada de Teller (1998).

Outros resultados mostraram possibilidade de discriminações no eixo tritan aos 2 meses de vida (Clavadetscher, Brown, Ankrum, \& Teller, 1988; Varner, Cook, Schneck, McDonald, \& Teller, 1985), mas, segundo Teller (1998), em experimentos subseqüentes com outras condições de estimulação 
(Dobkins \& Teller, observações não publicadas) houve dificuldade nessas discriminações, perdurando a dúvida sobre a prontidão do sistema azul-amarelo aos 2 meses.

Outro procedimento usado para estudar visão de cores em bebês foi o de Adams e colaboradores (Adams, Courage, \& Mercer, 1991), no qual era medida a resposta comportamental de habituação. Diante de um estímulo uniforme e outro de maior complexidade - uma grade - o bebê ficará mais tempo olhando para o estímulo mais complexo. Entretanto, se as listras da grade estiverem abaixo da capacidade de resolução do bebê, o tempo de inspeção dos dois estímulos não diferirá. Adams e colaboradores examinaram 40 recémnascidos com poucos dias de vida (média 3.2 dias) e concluíram que eles tinham a capacidade de discriminar um estímulo laranja $(595 \mathrm{~nm})$, mas não um estímulo verde-amarelado (565 nm), em relação a estímulos acromáticos. As dificuldades de discriminação incluem não só os comprimentos de onda médios (verde-amarelado), mas também os curtos (azul), e são atribuídas pelos autores a uma imaturidade dos cones $\mathrm{S}$ ou do sistema de oponência cromática azul-amarelo.

Um procedimento recente para evidenciar discriminação é o de novidade (Civan, Teller, \& Palmer, 2003). Comparando esse novo procedimento com o de preferência espontânea desenvolvido pelo grupo de Teller, os autores argumentam que, embora a preferência espontânea seja indicativa de discriminação, sua ausência não indica necessariamente incapacidade de discriminar. Preferência pela novidade pode ocorrer mesmo quando não há preferência espontânea. Nesse procedimento, os bebês são familiarizados com dois estímulos cromáticos idênticos e testados frente a uma mudança na cor de um dos dois estímulos. Se a mudança de cor resultar em preferência, a interpretação é de que houve discriminação. Civan et al. (2003) mediram a preferência espontânea para estímulos azuis e vermelhos em bebês de 16 semanas de vida. Em seguida, compararam o desempenho no teste de preferência pela novidade para os estímulos associados com alta preferência espontânea com os de pouca preferência espontânea. Os resultados mostraram que, na ausência de preferência espontânea, a preferência pela novidade pode ser usada para revelar discriminação. 


\title{
Visão de Cores no Primeiro Ano de Vida
}

Em conclusão, os métodos comportamentais permitiram investigar a visão de cores de bebês de forma não-invasiva, revelando a utilização do potencial neurológico detectado pelos métodos eletrofisiológicos. Em conjunto, esses estudos mostraram que as discriminações de cores mais simples são possíveis com o sistema de oponência verde-vermelho desde os dois meses de idade. Há resultados conflitantes entre os trabalhos sobre o desenvolvimento do sistema de oponência azul-amarelo - alguns estudos encontram discriminação aos 2 meses, outros apenas mais tarde. Estudos a partir dos 4 meses mostram que a detecção de contrastes de luminância e cromáticos revela maturação mais rápida para os contrastes de luminância do que para os cromáticos.

Agradecimentos: $\mathrm{O}$ apoio financeiro para a montagem e o funcionamento do Laboratório da Visão: Psicofísica e Eletrofisiologia Visual Clínica provém de projetos apoiados pela FAPESP (Projeto Temático 02/12733-8), pelo FINEP, pelo CNPq e pela CAPES (PROCAD). A autora é bolsista $1 \mathrm{~A}$ do $\mathrm{CNPq}$.

Ventura, D. F. (2007). Color vision in the first year of life. Psicologia USP, 18(2), 83-97.

\begin{abstract}
Behavioral procedures that allow the estimation of sensory or perceptual abilities in infants and children are greatly based on the seminal discovery made in the 1950 s by psychologist Robert Fantz that, given the choice, birth infants prefer to look at complex stimuli rather than to gaze at monotonous scenes. Based on the new discovery, the group of scientists led by Davida Teller, at the University of Washington, developed a methodology for psychophysical assessment of vision in babies that allowed a great advancement in the study of development and maturation of several basic visual functions, such as visual acuity, color vision, contrast sensitivity, stereoscopic vision, and vision of movement. The present review examines this literature showing how visual functions are assessed in babies and what has been learnt so far about the capacity to see color by the newborn.
\end{abstract}

Index terms: Color discrimination. Development. Infants. 


\section{Dora Fix Ventura}

Ventura, D. F. (2007). Vision de couleur dans la première année de vie. Psicologia USP, 18(2), 83-97.

Résumé: Les procédures comportementales qui permettent de juger les capacités sensorielles ou perceptuelles chez les bébés et les enfants sont fort basées sur la découverte du psychologue Robert Fantz, parue à la fin des années 1950. Dès la naissance les bébés préfèrent regarder les objets en mouvement plutôt que les scènes monotones. Le groupe de scientifiques dirigé par le Professeur Davida Teller, à l'Université de Washington, a développé une méthode d’évaluation psychophisique de la vision des bébés, ce qui a permis un avancement des études sur la maturation des fonctions visuelles. Une revue de la littérature nous permet de discuter cette méthode et d'aborder les connaissances actuelles sur la capacité de discrimination des couleurs chez les nouveau-nés.

Mots-clés: Discrimination de couleur. Développement. Bébés.

\section{Referências}

Adams, R. J., Courage, M. L., \& Mercer, M. E. (1991). Deficiencies in human neonates color-vision - photoreceptoral and neural explanations. Behavioural Brain Research, 43(2), 109-114.

Bornstein, M. H. (1975). Qualities of color-vision in infancy. Journal of Experimental Child Psychology, 19(3), 401-419.

Boynton, R. (1979). Human color vision. New York: Holt, Rinehart, and Winston.

Civan, A. L., Teller, D. Y., \& Palmer, J. (2003). Infant color vision: Spontaneous preferences versus novelty preferences as indicators of chromatic discrimination among suprathreshold stimuli. Journal of Vision, 3(9), 712a. Recuperado em 25 de dezembro, 2005, de http://journalofvision.org/3/9/712/, doi:10.1167/3.9.712

Clavadetscher, J. E., Brown, A. M., Ankrum, C., \& Teller, D. Y. (1988). Spectral sensitivity and chromatic discriminations in 3- and 7-week-old human infants. Journal of the Optical Society of America A-Optics Image Science and Vision, 5, 2093-2105.

Dobson, V. (1976). Spectral sensitivity of 2-month infant as measured by visually evoked cortical potential. Vision Research, 16(4), 367-374.

Fantz, R. L. (1956). A method for studying early visual development. Perceptual and Motor Skills, 6(1), 13-15.

Fantz, R. L. (1958). Pattern vision in young infants. Psychological Record, 8(2), 43-47. 


\section{Visão de Cores no Primeiro Ano de Vida}

Fantz, R. L. (1960). A method for studying depth-perception in infants under 6-months of age. American Psychologist, 15(7), 480.

Fantz, R. L. (1961). A method for studying depth-perception in infants under 6 months of age. Psychological Record, 11(1), 27-32.

Fantz, R. L. (1963). Pattern vision in newborn infants. Science, 140(356), 296-297

Fantz, R. L. (1964). Visual experience in infants - decreased attention to familiar patterns relative to novel ones. Science, 146(364), 668-670

Fantz, R. L. (1965). Visual perception from birth as shown by pattern selectivity. Annals of the New York Academy of Sciences, 118(A21), 793-814.

Fantz, R. L., \& Miranda, S. B. (1975). Newborn-infant attention to form of contour. Child Development, 46(1), 224-228.

Fantz, R. L., \& Nevis, S. (1967). Pattern preferences and perceptual-cognitive development in early infancy. Merrill-Palmer Quarterly of Behavior and Development, 13(1), 77-108.

Fantz, R. L., \& Ordy, J. M. (1959). A visual-acuity test for infants under 6 months of age. Psychological Record, 9(4), 159-164.

Fantz, R. L., Ordy, J. M., \& Udelf, M. S. (1962). Maturation of pattern vision in infants during first 6 months. Journal of Comparative and Physiological Psychology, 55(6), 907-917.

Hamer, R. D., Alexander, K. R., \& Teller, D. Y. (1982). Rayleigh discriminations in young human infants. Vision Research, 22(5), 575-587.

Kelly, J. P., Borchert, K., \& Teller, D. Y. (1997). The development of chromatic and achromatic contrast sensitivity in infancy as tested with the sweep VEP. Vision Research, 37(15), 2057-272.

Kelly, J. P., \& Chang, S. (2000). Development of chromatic and luminance detection contours using the sweep VEP. Vision Research, 40(14), 1887-1905.

Kessen, W., \& Bornstein, M. H. (1978). Discriminability of brightness change for infants. Journal of Experimental Child Psychology, 25(3), 526-530.

Knoblauch, K., Bieber, M. L, \& Werner, J. S. (1998). M- and L-cones in early infancy: I. VEP responses to receptor-isolating stimuli at 4- and 8-weeks of age. Vision Research, 38(12), 1753-1764.

Knoblauch, K., Vital-Durand, F., \& Barbur, J. L. (2001). Variation of chromatic sensitivity across the life span. Vision Research, 41(1), 23-36.

Lee, B. B. (1997). Parallel pathways in primate retina. In C. Dickinson, I. Murray \& D. Carden (Eds.), John Dalton's colour vision legacy (pp. 24-25) Manchester, UK: Taylor and Francis. 


\section{Dora Fix Ventura}

MacLeod, D. I. A., \& Boynton, R. M. (1979). Chromaticity diagram showing cone excitation by stimuli of equal luminance. Journal of the Optical Society of America, 69, 11831186.

Merigan, W. H., \& Maunsell, J. H. R. (1993). How parallel are the primate visual pathways? Annual Review of Neuroscience, 16, 369-402.

Morrone, M. C., Burr, D. C., \& Fiorentini, A. (1993). Development of infant contrast sensitivity to chromatic stimuli. Vision Research, 33(17), 2535-2552.

Morrone, M. C., Fiorentini, A., \& Burr, D. C. (1996). Development of the temporal properties of visual evoked potentials to luminance and colour contrast in infants. Vision Research, 36(19), 3141-3155.

Oliveira, A. G. F., Costa, M. F., De Souza, J. M., \& Ventura, D. F. (2004). Contrast sensitivity threshold measured by sweep-visual evoked potential in term and preterm infants at 3 and 10 months of age. Brazilian Journal of Medical and Biological Research, 37(9), 1389-1396.

Peeples, D. R., \& Teller, D. Y. (1975). Color-vision and brightness-discrimination in 2 month-old human infants. Science, 189(4208),1102-1103.

Peeples, D. R., \& Teller, D. Y. (1978). White-adapted photopic spectral sensitivity in human infants. Vision Research, 18(1), 49-53.

Salomão, S. R., \& Ventura, D. F. (1993). The Brazilian Large-Scale Acuity Card Study .1. The normative study. Investigative Ophthalmology \& Visual Science, 34(4), 1420.

Salomão, S. R., \& Ventura, D. F. (1995). Large-Sample Population Age Norms for Visual Acuities Obtained with Vistech-Teller Acuity Cards. Investigative Ophthalmology \& Visual Science, 36(3), 657-670.

Schaller, M. J. (1975). Chromatic vision in human infants - conditioned operant fixation to hues of varying intensity. Bulletin of the Psychonomic Society, 6(1), 39-42.

Teller, D. Y. (1998). Spatial and temporal aspects of infant color vision. Vision Research, 38, 3275-3282.

Teller, D. Y., Peeples, D. R., \& Sekel, M. (1978). Discrimination of chromatic from whitelight by 2-month-old human infants. Vision Research, 18(1), 41-48.

Varner, D., Cook, J. E., Schneck, M. E., McDonald, M., \& Teller, D. Y. (1985). Tritan discriminations by 1- and 2-month-old human infants. Vision Research, 25, 821-831.

Ventura, D. F., Rodrigues, A. R., Moura, A. A., Vargas, A. C., Costa, M. F., de Souza, J. M. et al. (2002). Color discrimination measured by the cambridge colour vision test (CCNT) in children and adults. Investigative Ophthalmology \& Visual Science, 43, U1046. 


\section{Visão de Cores no Primeiro Ano de Vida}

Xiao, M., \& Hendrickson, A. (2000). Spatial and temporal expression of short, long/medium, or both opsins in human fetal cones. Journal of Comparative Neurology, 425(4), 545559.

Recebido em: 3.07.2007

Aceito em: 6.08.2007 
